\title{
Scanning force microscopy in the dynamic mode using microfabricated capacitive sensors
}

\author{
N. Blanc, ${ }^{\text {a) }}$ J. Brugger, ${ }^{\text {b) }}$ and N. F. de Rooij \\ Institute of Microtechnology (IMT), University of Neuchâtel, Jaquet-Droz 1, 2007 Neuchâtel, Switzerland \\ U. Dürig \\ IBM Research Division, Zurich Research Laboratory, 8803 Rüschlikon, Switzerland
}

(Received 25 July 1995; accepted 18 December 1995)

\begin{abstract}
We report on the first successful operation of a scanning force microscope using microfabricated capacitive force sensors. The sensors, which are made from single crystal silicon on insulator wafers, consist of a cantilever spring with integrated tip at the free end and an electrically insulated counter electrode. Dynamic force gradient sensing is the preferred operating mode. Here, tipsample interactions are detected by letting the sensor act as a resonator in a phase controlled oscillator setup and measuring corresponding shifts of the oscillation frequency. Experiments were performed in vacuum using a standard tunneling microscope. A Cr grating on a quartz substrate served as the test sample. Topographic images showing details on a $10 \mathrm{~nm}$ scale were obtained operating at a constant force gradient of the order of $0.01 \mathrm{~N} / \mathrm{m}$. In addition, critical design parameters are discussed based on an analysis of the electromechanical properties of the sensors.

(C) 1996 American Vacuum Society.
\end{abstract}

\section{INTRODUCTION}

Prospects for making miniaturized stand-alone scanning force microscopes have triggered the development of various force sensors with integrated detection schemes such as piezoresistive $^{1}$ or piezoelectric cantilevers. ${ }^{2,3}$ Compared to optical detection these sensors have a much smaller overall size and do not require any critical alignment of external components. Capacitive detection is also an interesting and very sensitive technique that is commonly applied in the field of pressure sensors and accelerometers. A major advantage of capacitive detectors is the fact that they contain only reactive components and therefore they are free of parasitic resistive noise sources that adversely affect the sensitivity. In the field of scanning force microscopy (SFM) various groups have already reported interesting results based on assembled capacitive setups. ${ }^{4,5}$ In this article we present capacitive sensors manufactured by batch processing techniques. Each sensor combines an integrated capacitive detection of the cantilever displacement as well as an electrostatic actuation. ${ }^{6-8}$ This property makes capacitive sensors particularly attractive for applications requiring parallel operation of large sensor arrays, e.g., as is envisioned in high-density data storage devices based on local probe techniques. The fabrication process of these novel devices is based on bulk silicon micromachining of silicon on insulator (SOI) wafers. The electromechanical characteristics of the capacitive microlevers are discussed and topographic images of a chrome grating are presented which were obtained in a dynamic operating mode regulating on a constant force gradient.

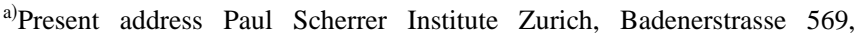
CH-8048 Zurich, Switzerland.

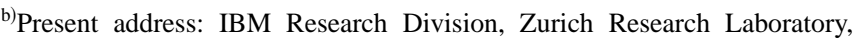
8803 Rüschlikon, Switzerland.

\section{STRUCTURE DESIGN AND FABRICATION}

The design of the sensors is schematically shown in Fig. 1. They consist of two adjacent, highly doped single-crystal silicon beams forming the two plates of a capacitor. One of the beams, called the cantilever (CB), is fairly soft and it serves as a spring for sensing interaction forces. A tip is located at the free end of the CB. Typical CB dimensions are: width $w=60 \mu \mathrm{m}$, thickness $t=5-6 \mu \mathrm{m}$, and length $l$ ranging from 275 to $350 \mu \mathrm{m}$. The second beam, termed counter electrode, is essentially rigid and its typical dimensions are: $w=80 \mu \mathrm{m}, t=15 \mu \mathrm{m}$, and $l$ is in the same range as the corresponding $\mathrm{CB}$. The cantilever spring constant and resonant frequency are computed according to

$$
k=\frac{3 E I}{l^{3}}
$$

and

$$
f=0.162 t / l^{2}(E / \rho)^{1 / 2},
$$

with $I=w t^{3} / l^{2}$ the moment of inertia, $E=1.7 \times 10^{11} \mathrm{~N} / \mathrm{m}^{2}$ Young's modulus, and $\rho=2300 \mathrm{~kg} / \mathrm{m}^{3}$ the density of silicon. The spring constant and resonant frequency range from 7 to $26.5 \mathrm{~N} / \mathrm{m}$ and $57-111 \mathrm{kHz}$, respectively. Note that holes have been patterned into the cantilevers in order to promote etching of the thin silicon dioxide layer in the fabrication of the gap between the cantilever and counter electrode [see Fig. 2(g)]. As a result of the holes, the resonance frequency and the spring constant of the sensors are somewhat smaller than the corresponding values computed by means of Eqs. (1) and (2). The tip can be actuated electrostatically, as described in Ref. 8. For low applied voltages $V$ the displacement is

$$
z_{0}=\frac{1}{16} \epsilon_{0} \frac{V^{2}}{d^{2}} \frac{w l^{4}}{E I},
$$




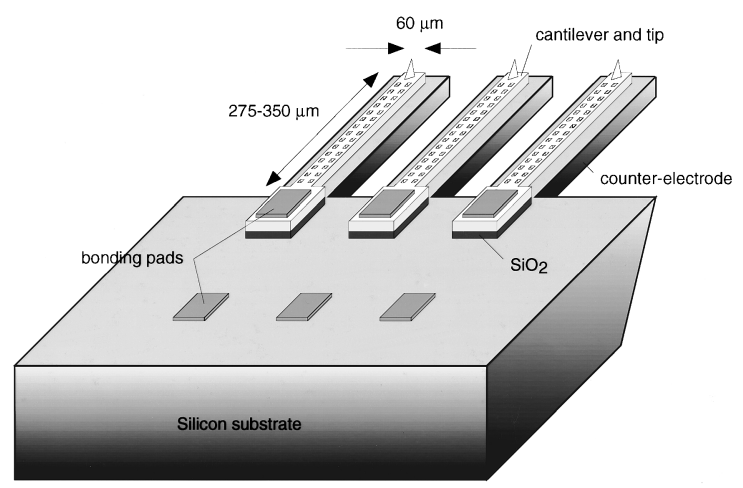

FIG. 1. Schematic outline of the capacitive sensor for scanning force microscopy. The cantilever and its counter electrode are separated by a small gap, typically $1 \mu \mathrm{m}$ wide, and they are attached to a Si holder containing bonding pads for electrical contacts to the lever and the counter electrode.

with $\epsilon_{0}=8.85 \times 10^{-12} \mathrm{As} / \mathrm{Vm}$ the permittivity in vacuum and $d=1 \mu \mathrm{m}$ the gap between the cantilever and counter electrode. Equation (3) can be written as $z_{0}=\alpha V^{2}$ and for the present cantilever dimensions one obtains an $\alpha$ value of the order of $1 \mathrm{~nm} / \mathrm{V}^{2}$.

The devices are fabricated by silicon bulk micromachining. The process is illustrated in Fig. 2. SOI wafers with a 15 - $\mu$ m-thick $n$-type (100) oriented silicon film, a $1-\mu \mathrm{m}$-thick buried $\mathrm{SiO}_{2}$ layer, and a $400-\mu$ m-thick $n$-type (100) oriented silicon substrate were used [see Fig. 2(a)]. The resistivity of the $\mathrm{Si}$ was of the order of $10 \Omega \mathrm{cm}$ which turned out to be somewhat high for sensor applications (see below). Silicon dioxide is first thermally grown to a thickness of $1.7 \mu \mathrm{m}$ on both sides. Three photolithographic and subsequent $\mathrm{SiO}_{2}$ etching steps in buffered hydrofluoric acid (BHF) are used to pattern the oxide mask and define the cantilevers and tips as well as the backside etch windows [see Fig. 2(b)]. The counter electrode is etched by reactive ion etching (RIE) with photoresist as a mask. The buried oxide spacer layer between the $\mathrm{CB}$ and the counter electrode is then removed by BHF etching [see Fig. 2(c)] and the photoresist is stripped in acetone. The cantilevers with integrated tips are defined by

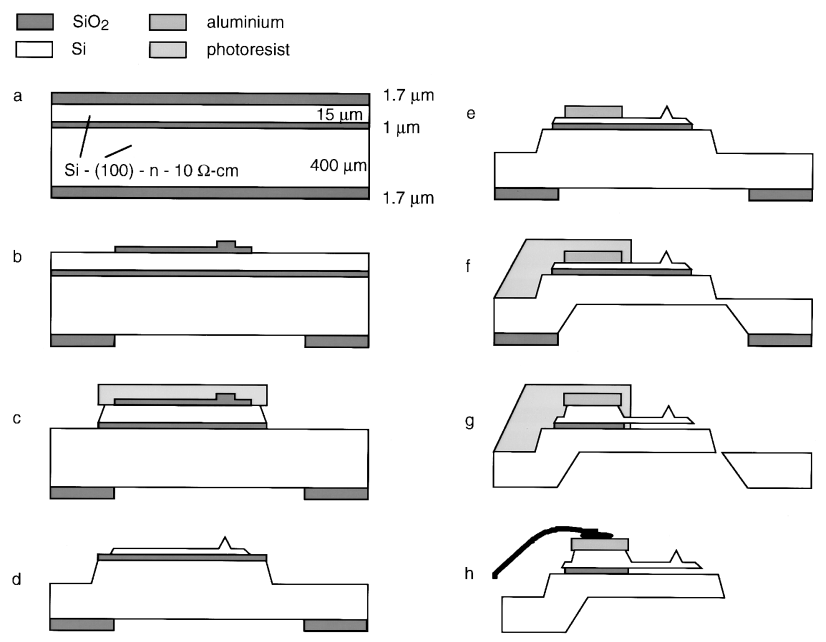

FIG. 2. Process steps in the microfabrication of the capacitive microlevers. successive RIE and BHF etchings as previously reported for simple cantilevers ${ }^{9}$ [see Fig. 2(d)]. In order to lower the resistivity of the cantilever structure a phosphorus-doped silicate glass (PSG) is deposited, followed by a diffusion step and the removal of the PSG layer in BHF.

A $1-\mu \mathrm{m}$-thick layer of aluminum is then deposited, patterned, and alloyed for $30^{\prime}$ at $450{ }^{\circ} \mathrm{C}$ to form the ohmic contacts to the cantilevers and the substrate [see Fig. 2(e)]. A membrane, typically $30 \mu \mathrm{m}$ thick, is defined by anisotropic etching in $\mathrm{KOH}$ from the backside while protecting the top side in a mechanical chuck [see Fig. 2(f)]. An appropriately patterned photoresist cover layer protects the aluminum bonding pads from being attacked in the subsequent fabrication steps. The counter electrodes and the cantilevers are released by RIE from the backside and BHF etching, respectively [see Fig. 2(g)]. Finally, the resist is stripped and the cantilevers bonded [see Fig. 2(h)]. Figure 3 shows micrographs of the microfabricated capacitive cantilevers after the completion of the process.

\section{EXPERIMENTAL RESULTS}

The frequency response of the electrical impedance of the capacitive sensor deviates significantly from the one of a simple capacitor due to the coupling of the mechanical motion of the $\mathrm{CB}$ and corresponding electrical fields. The frequency response can be modeled by the same equivalent circuit that applies to a quartz crystal resonator. It consists of a series resonant circuit that is shunted by a capacitance. ${ }^{10} \mathrm{~A}$ detailed analysis shows ${ }^{11}$ that observability of the resonant properties and the overall performance of the capacitive sensor can be expressed in terms of a single parameter that is to be maximized and which scales as the square of the applied biasing voltage and the inverse cube of the gap width between CB and counter electrode. Hence, the quality of the insulating oxide layer and of the capacitor gap are crucial for successful operation of capacitive sensors.

For practical reasons the admittance which is the inverse of the impedance was measured using a spectrum analyzer (see Fig. 4). For this purpose a small (of the order of $5 \mathrm{mV}$ ) white noise driving voltage $\mathrm{e}^{7,8}$ superimposed on a dc bias voltage ( $36 \mathrm{~V}$ in the example shown) is applied. The current that is induced in the capacitive sensor is measured by means of a virtual ground type current to voltage converter. By this scheme the electrical potential of the cantilever (and the tip) is clamped to ground potential thereby virtually suppressing effects due to unavoidable stray capacitances. This point is somewhat critical as the capacitance of the lever is only of the order $0.1 \mathrm{pF}$. The frequency response of admittance measured on a cantilever with a length of $300 \mu \mathrm{m}$ is shown in Fig. 5. Series and parallel resonance can be clearly identified at $f_{s}=60147.5 \mathrm{~Hz}$ and $f_{p}=60217.5 \mathrm{~Hz}$, respectively. As expected, a sharp transition in the phase response is observed. The phase shift amounts to $160^{\circ}$ and the slope measured at the series resonance is $\partial \phi / \partial f)_{f=f s}=9^{\circ} / \mathrm{Hz}$ from which a quality factor of the resonance of the order of $Q=f / 2 \partial \phi / \partial f)_{f=f_{s}}=4700$ can be deduced. The observed $Q$ factor appears to be somewhat low. The reason for this is the 
a

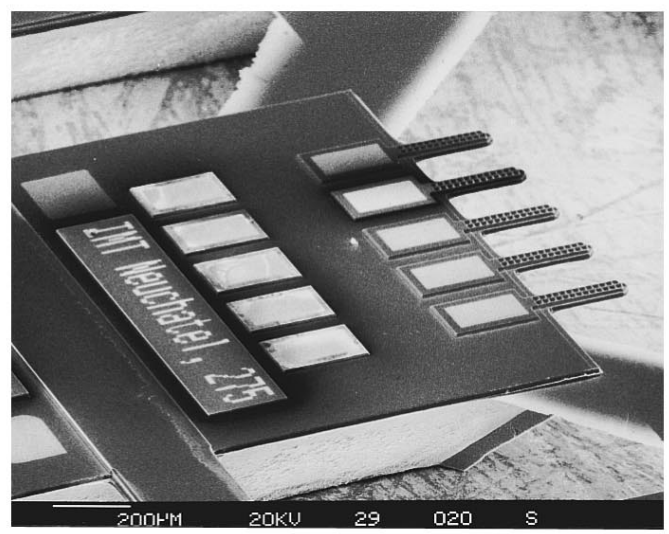

b

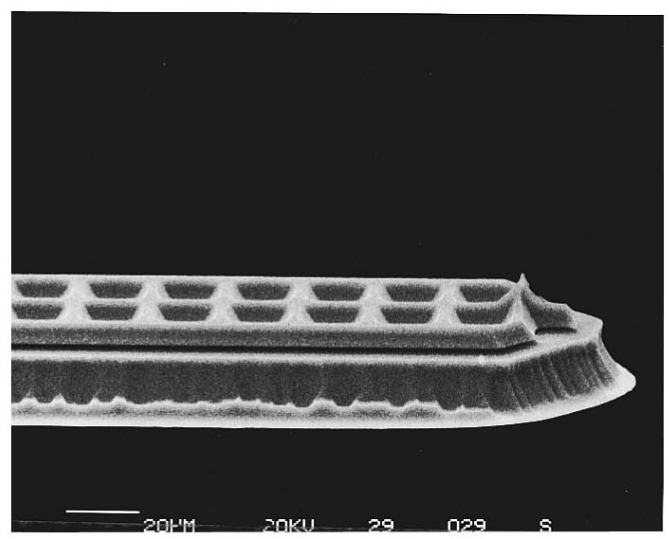

C

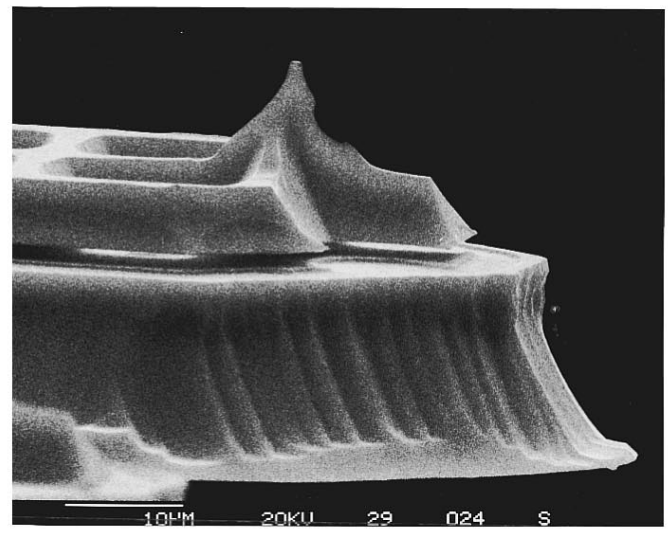

FIG. 3. (a) SEM micrograph of an array of micromachined capacitive cantilever sensors. (b), (c) Enlarged views of the end section of the lever showing the integrated probe tip.

high value of the specific resistance of the Si wafers used. The $Q$ factor is lowered because of resistive dissipation induced by the motion of space charge at the surface of the CB and the counter electrode when the electric field is being modulated by the vibration of the CB. Quality factors as high as 50000 have been obtained in test devices that were doped close to the limit of degeneracy.

With increasing applied dc voltage the resonant frequency decreases, as shown in Fig. 6. This corresponds to a lowering of the effective cantilever spring constant. At low applied dc bias the frequency shift is proportional to the voltage squared. From this data the mechanical resonant frequency (at $V_{\mathrm{dc}}=0$ ) is extrapolated to be $66585 \mathrm{~Hz}$. The physical

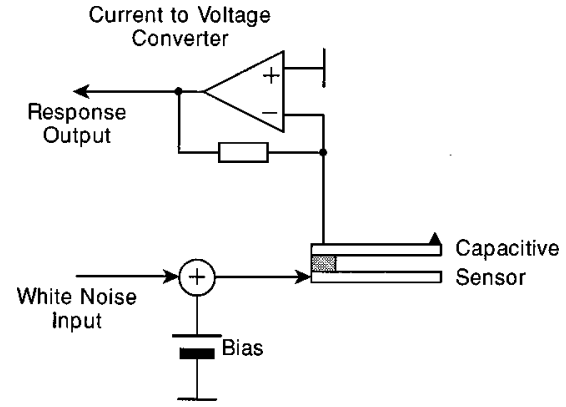

FIG. 4. Schematic diagram of the setup used in the admittance measurement. Note that the CB is virtually grounded by means of the current to voltage converter and hence the effect of stray capacitances is minimized.

dimensions of the cantilevers were checked by scanning electron microscopy. The observed thickness of the levers varied between 5 and $6 \mu \mathrm{m}$. From Eq. (2) we compute a resonance frequency in the range $77-93 \mathrm{kHz}$ for cantilevers with a length of $300 \mu \mathrm{m}$. These values are higher than the experimentally determined resonance frequency. This difference is attributed to the effect of the holes in the cantilever. Note that due to thickness variation the measured resonance frequency itself also varies by a substantial amount. Values as high as $85 \mathrm{kHz}$ have been observed for the type of cantilevers used in the experiments.

Dynamic force gradient sensing is the preferred operating mode for capacitive force sensors. The method exploits the fact that the resonance frequency of the CB shifts according to the interaction force gradient acting between the tip and
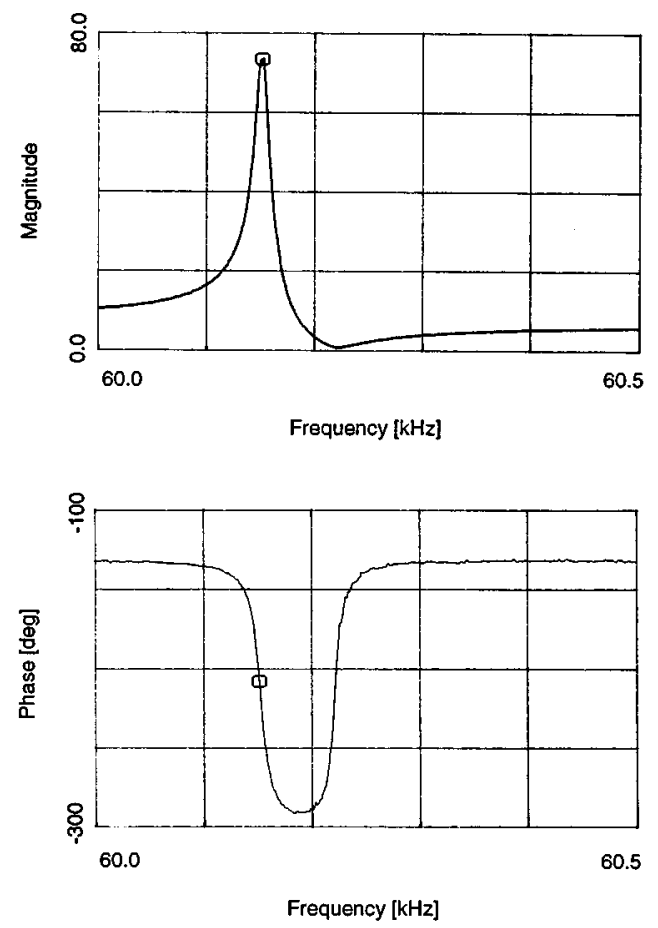

FIG. 5. Measured frequency response (admittance amplitude and phase) of a capacitive cantilever with a length of $300 \mu \mathrm{m}$. The applied bias is $V_{\mathrm{dc}}=36 \mathrm{~V}$. 


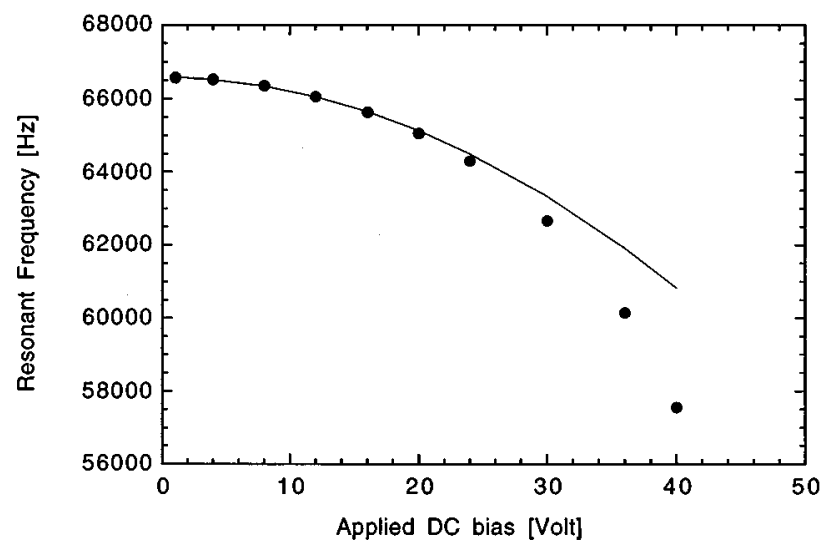

FIG. 6. Measured series resonant frequency as a function of applied dc bias for a cantilever with a length of $300 \mu \mathrm{m}$. The solid line corresponds to $f_{s}=66585-3.6 V_{\mathrm{dc}}^{2}$.

the sample. Implementation of the scheme is straightforward as the capacitive sensor acts like a resonant electrical twoterminal device. For measuring the resonance frequency a setup similar to the one used for the admittance measurement was employed (see Fig. 7). The sensor serves as the frequency determining element (operating at series resonance) in an oscillator circuit that consists of a variable gain feedback amplifier and an automatic gain control to maintain a constant vibration amplitude. ${ }^{12}$

The oscillation frequency is measured by means of a phase-locked loop frequency detector that has a bandwidth of $10 \mathrm{kHz}$.

Experiments were performed in vacuum in order to avoid damping of the CB oscillation by ambient air. A dc bias of 10 $\mathrm{V}$ was applied and an ac driving voltage of the order of $5 \mathrm{mV}$ was sufficient to induce a vibration amplitude of $50 \mathrm{~nm}$ at the free end of the cantilever. A Cr grating on a quartz substrate

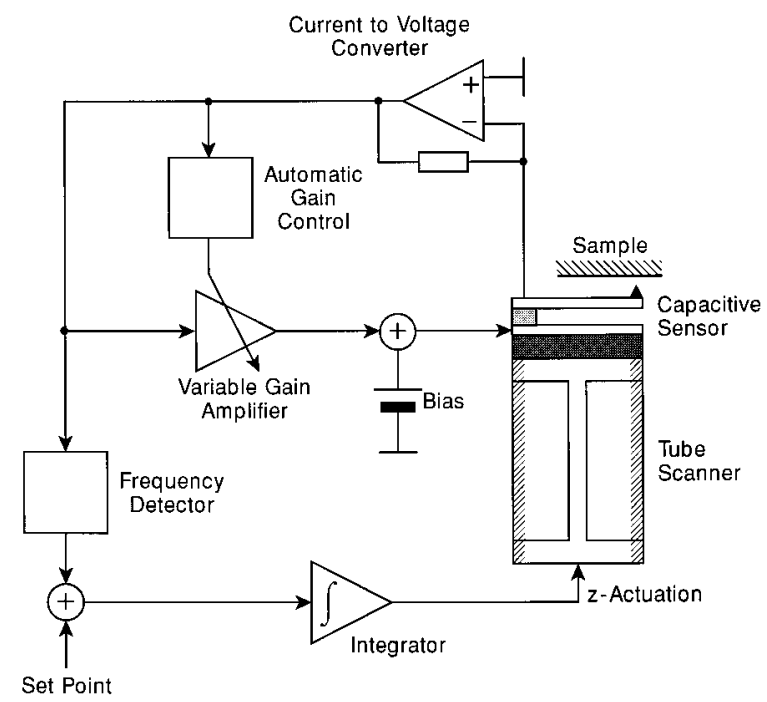

FIG. 7. Schematic diagram of the oscillator and the associated feedback control loop which controls the tip-sample distance by regulating on a constant frequency shift.
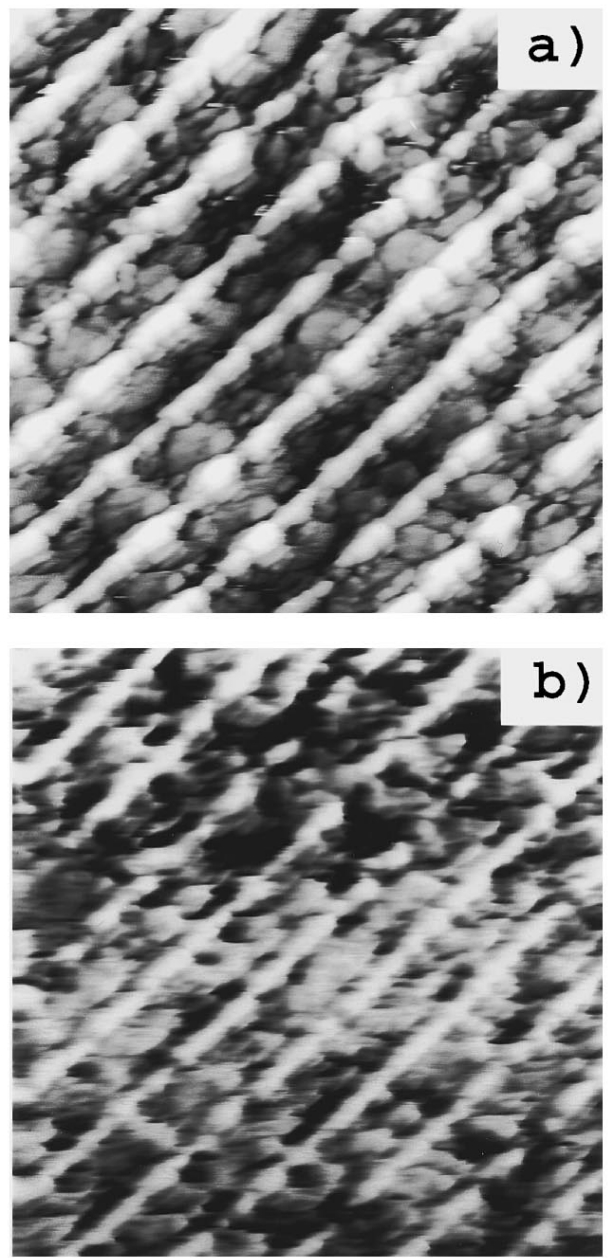

FIG. 8. Dynamic force gradient imaging of a $\mathrm{Cr}$ grating with a pitch of 250 $\mathrm{nm}$ on a quartz substrate. The total scan area is $1.5 \mu \mathrm{m} \times 1.5 \mu \mathrm{m}$. The image was obtained in the repulsive ("tapping") mode at an effective positive force gradient of $0.01 \mathrm{~N} / \mathrm{m}$. (a) Topography: height variation between trenches, shown dark, and ridges, shown bright, corresponds to $80 \mathrm{~nm}$. (b) Dissipation: low and high dissipation are shown as bright and dark tones, respectively. The dissipation in the dark areas is about two times stronger than in the bright areas.

was used as test sample. The pitch of the grating was $250 \mathrm{~nm}$ and the $\mathrm{Cr}$ lines had a height of $100 \mathrm{~nm}$. Topographic imaging was performed in a constant force gradient operating mode meaning that the feedback loop controlling the tipsample distance was regulating on a constant frequency shift. At these conditions the noise of the output of the frequency detector was of the order of $2 \mathrm{~Hz}$ measured over a bandwidth of $1 \mathrm{kHz}$. This noise level is roughly a factor of 2 larger than the fundamental thermal oscillator noise limit. ${ }^{12}$ A positive shift of $50 \mathrm{~Hz}$ was selected as imaging parameter in Fig. 8(a) showing a $1.5 \mu \mathrm{m} \times 1.5 \mu \mathrm{m}$ section of the grating. The frequency shift translates into a positive interaction force gradient of the order of $0.01 \mathrm{~N} / \mathrm{m}$ (assuming a spring constant of $10 \mathrm{~N} / \mathrm{m}$ ) meaning that the overall tip-sample interaction was repulsive. In fact, operation of the force microscope is better described in terms of a high-frequency tapping mode given that the vibration amplitude of the $\mathrm{CB}$ was of the order of 50 $\mathrm{nm}$. In each oscillation cycle the tip pushes against the 
sample for a short fraction of the oscillation period. The repulsive force that was exerted by the tip during touching can be estimated to be of the order of $500 \mathrm{pN}$ assuming $1 \mathrm{~nm}$ for the range of interaction. The drive voltage necessary to maintain a constant amplitude of vibration is a measure for dissipation. ${ }^{12}$ By monitoring this voltage [see Fig. 8(b)], one can thus obtain information on local variations of dissipative channels of the tip-sample interaction. Correlation of the topography with corresponding variations of the damping factor can easily be recognized. Damping forces are highest when the tip is following a trench between $\mathrm{Cr}$ lines and they are smallest when the tip is on top of a $\mathrm{Cr}$ line. At this point we lack a firm understanding of the physical mechanism that is causing this variation of the dissipative interaction. From the correlation with the topography, namely, dissipation being minimum on top of the protrusions and maximum at the edges of the ridges, one might speculate that the dissipation is caused by frictional forces as the tip is touching the sample surface.

To conclude, we have shown that microfabricated capacitive sensors can be used for scanning force microscopy in the dynamic mode. With this device the experimental setup can be greatly simplified with respect to many of the conventional schemes: no optical components for the tip displacement sensing are needed and, moreover, as shown in this article, the integrated electrostatic actuation can be used to excite the levers at resonance, meaning that no additional actuators are required. Static actuation is also conceivable. However, one must keep in mind that the electrostatic potential applied to the actuator also influences its resonance frequency. Therefore, compensation schemes are needed in or- der to separate this effect from genuine interaction induced changes of the resonance frequency.

\section{ACKNOWLEDGMENTS}

The authors wish to thank C. Ketterer and P. Sixt from CSEM for performing the SEM studies and providing the $\mathrm{Cr}$ grating, respectively. Technical assistance from P. A. Clerc and S. Pochon (IMT), from P. Lacombe and J.-D. Cretin (Microsens), and from T. Mezzacasa (PSI) is gratefully acknowledged. This work was supported by the Swiss National Science Foundation.

${ }^{1}$ M. Tortonese, H. Yamada, R. C. Barrett, and C. F. Quate, in The Proceedings of Transducers ' 91 (IEEE, Pennington, NJ, 1991), Publ. No. 91 CH2817-5, p. 448.

${ }^{2}$ T. Itoh and T. Suga, Sensors Actuators A 43, 305 (1994).

${ }^{3}$ S. Akamine, T. R. Albrecht, M. J. Zdeblick, and C. F. Quate, Sensors Actuators A 21, 964 (1990).

${ }^{4}$ T. Goddenhenrich, H. Lemke, U. Hartmann, and C. Heiden, J. Vac. Sci. Technol. A 8, 383 (1990).

${ }^{5}$ G. Neubauer, S. R. Cohen, G. M. McClelland, D. Horne, and C. M. Mate, Rev. Sci. Instrum. 61, 2296 (1990).

${ }^{6}$ L. C. Kong, B. G. Orr, and K. D. Wise, J. Vac. Sci. Technol. B 11, 634 (1993).

${ }^{7}$ J. Brugger, N. Blanc, Ph. Renaud, and N. F. de Rooij, Sensors Actuators A 43, 339 (1994).

${ }^{8}$ N. Blanc, J. Brugger, and N. F. de Rooij, in Forces in Scanning Probe Methods, edited by H.-J. Guntherodt et al. [NATO ASI Ser. E: Appl. Sci. 286, 79 (1995)].

${ }^{9}$ J. Brugger, R. A. Buser, and N. F. de Rooij, Sensors Actuators A 34, 193 (1992).

${ }^{10}$ M. W. Putty, M.S. thesis, University of Michigan at Ann Arbor, 1988.

${ }^{11}$ N. Blanc and U. Duerig (to be published).

${ }^{12}$ T. R. Albrecht, P. Grütter, D. Horne, and D. Rugar, J. Appl. Phys. 69, 668 (1991); U. Dürig, O. Züger, and A. Stalder, ibid. 72, 1778 (1992). 